
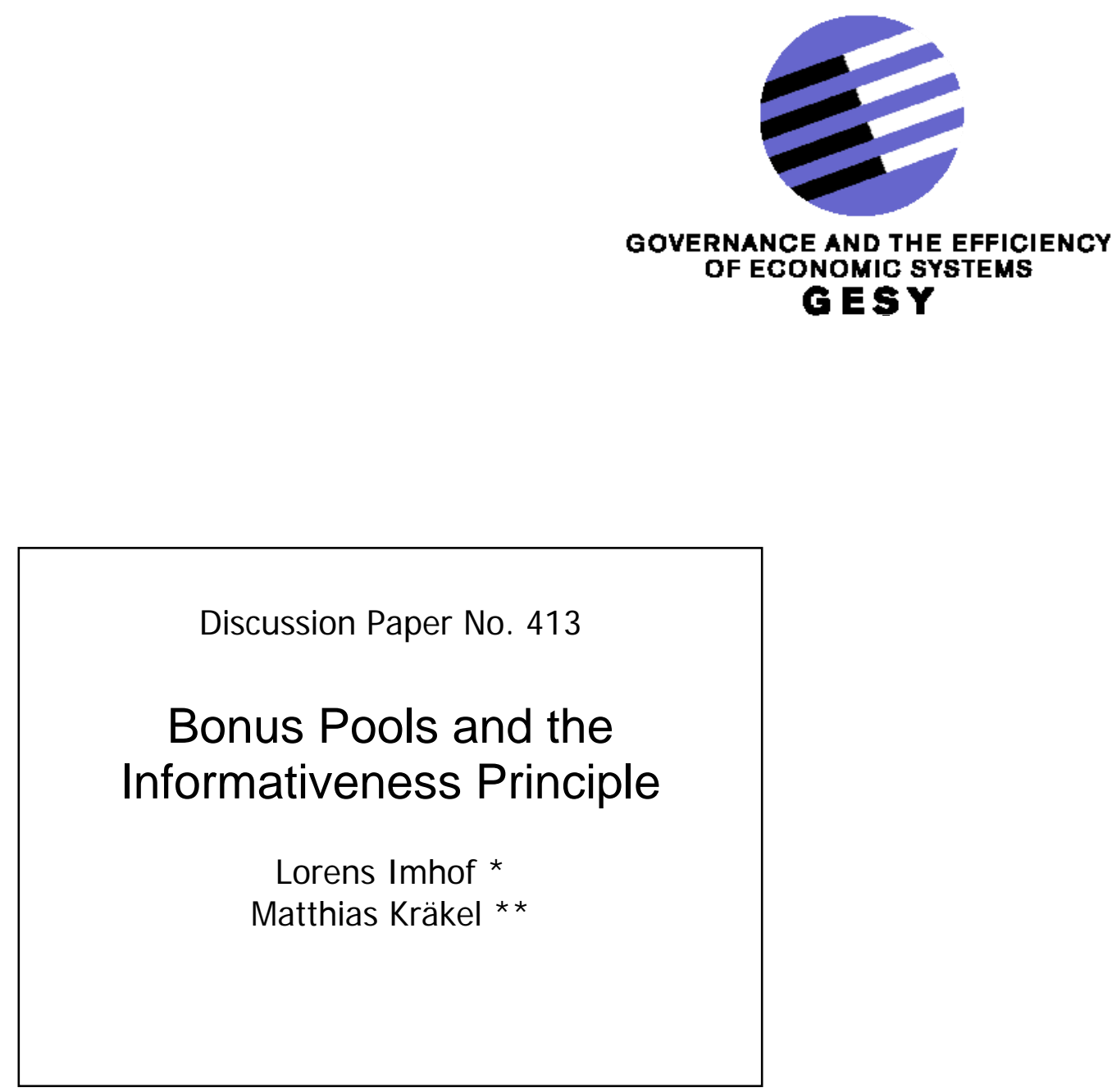

* University of Bonn

** University of Bonn

Financial support from the Deutsche Forschungsgemeinschaft through SFB/TR 15 is gratefully acknowledged.

Sonderforschungsbereich/Transregio $15 \cdot$ www.sfbtr15.de

Universität Mannheim · Freie Universität Berlin · Humboldt-Universität zu Berlin · Ludwiq-Maximilians-Universität München Rheinische Friedrich-Wilhelms-Universität Bonn · Zentrum für Europäische Wirtschaftsforschung Mannheim 


\title{
Bonus Pools and the Informativeness Principle*
}

\author{
Lorens Imhof, University of Bonn** \\ and \\ Matthias Kräkel, University of Bonn***
}

\begin{abstract}
Previous work on moral-hazard problems has shown that, under certain conditions, bonus contracts create optimal individual incentives for risk-neutral workers. In our paper we demonstrate that, if a firm employs at least two workers, it may further benefit from combining worker compensation via a bonus-pool contract and relative performance evaluation. Such combination leads to saved rents under a wide class of luck distributions. In addition, if the employer is wealth-constrained, complementing individual bonus contracts by the possibility of pooling bonuses can increase the set of implementable effort levels. All our results hold even though workers' outputs are technically and stochastically independent so that, in view of Holmstrom's informativeness principle, individual bonus contracts would be expected to dominate bonus-pool contracts.
\end{abstract}

Key words: contract; hazard rate; informativeness principle; limited liability; relative performance.

JEL classification: C72; D86.

* We would like to thank Jörg Budde, Florian Englmaier, Thomas Gall, Oliver Gürtler, Eugen Kovac, Daniel Krähmer, Benny Moldovanu, Daniel Müller, Klaus Sandmann, Urs Schweizer, Christian Seel, Tymon Tatur, the participants of the "Young Researchers' Workshop on Contests and Tournaments", in Dortmund, the participants of the annual conference of the German Economic Association (VfS), in Frankfurt, the participants of the GESY conference ("Governance and the Efficiency of Economic Systems") in Bonn, and the participants of the BGSE Micro Workshop for helpful comments. Financial support by the DFG, grant $\mathrm{SFB} / \mathrm{TR} 15$, is gratefully acknowledged.

** Lorens Imhof, Department of Economics and Hausdorff Center for Mathematics, University of Bonn, Adenauerallee 24-42, 53113 Bonn, Germany, phone: +49-228-739265, fax: +49-228-735050, e-mail: limhof@uni-bonn.de.

*** Matthias Kräkel, Department of Economics, University of Bonn, Adenauerallee 24-42, 53113 Bonn, Germany, phone: +49-228-739211, fax: +49-228739210, e-mail: m.kraekel@uni-bonn.de. 


\section{Introduction}

In a moral-hazard problem, incentives can be created by using either a carrot or a stick. In a world where workers are protected by limited liability, only carrots may be available. We show that the employer often can reduce rents paid to the worker by using one big carrot in the form of a bonus pool, instead of several small carrots that serve as individual bonus payments. Our results hold even when workers perform completely independent tasks. On the surface, this seems to contradict Holmstrom's informativeness principle ${ }^{1}$ that bonus pools are inferior to individual bonuses because relative performance information is noisier. When the firm faces constraints on the size of the bonuses it can pay out, however, the advantage of using one large carrot frequently outweighs the informational advantage of using several small carrots.

Individual bonus contracts are not only frequently observed in practice (e.g., Joseph and Kalwani 1998), but are also often optimal in a second-best setting (e.g., Demougin and Fluet 1998, Oyer 2000, Herweg et al. 2010). Bonus contracts specify a certain threshold or quota together with a wage premium that is paid to a worker if his output exceeds the given quota. If workers are risk neutral and the monotone likelihood ratio property holds, it is optimal for the employer to create high powered incentives by combining a very large quota with a very large wage premium.

In this paper, we analyze under which conditions a firm that employs at least two workers can benefit from pooling incentives. Under a bonus pool, the firm specifies a fixed amount of money to be distributed among the workers according to relative performance. In addition to the bonus payments, the bonus-pool contract specifies a minimum distance or gap by which one worker must outperform his co-workers to get a high bonus. ${ }^{2}$ This gap works similar as the quota of an individual bonus contract because it

\footnotetext{
${ }^{1}$ See Holmstrom $(1979,1982)$.

${ }^{2}$ Alternatively, we can speak of a tournament scheme that is complemented by a gap; see Nalebuff and Stiglitz (1983), pp. 30-32.
} 
influences both incentives and the probability with which bonus payments are made to the workers.

Since the workers' tasks are neither technically nor stochastically related, at first sight it does not make sense to pool workers' incentives: as relative performance is less informative about individual effort choice than absolute performance, individual incentives should always work better than collective ones according to Holmstrom's informativeness principle. In other words, compared to individual bonuses, a certain effort level can only be implemented via a bonus pool with relative performance evaluation if the bonus pool specifies a sufficiently larger wage premium. The extra money is needed to replace missing incentives stemming from the fact that the impact of individual effort on the performance measure is less strong. Hence, bonus pools should be too expensive and leave too much rents to the workers.

We offer two reasons why a bonus-pool contract can nevertheless beat individual bonus contracts: (1) in case of an increasing hazard rate, the employer prefers the highest possible threshold (together with large bonus payments) under both types of contract. If the thresholds are bounded above (e.g., due to a collective agreement between an industry wide union and an employer association), the employer will choose this upper bound for either contract. However, the effective threshold under a bonus pool with relative performance evaluation is considerably larger than the threshold under individual bonuses since under a bonus pool each worker has to beat the upper bound and his opponents which leads to a lower probability of paying a large bonus and, hence, to reduced worker rents. (2) Under a bonus pool, several workers compete for only one large bonus payment, whereas under individual bonuses each worker has to be incentivized by a separate bonus. If the employer is financially constrained, one big bonus pool may work better than several individual bonuses, which have to be rather small due to the employer's limited wealth. In addition to saving rents, pooling bonuses may increase the set of implementable efforts. We show that this second feature of bonus pools is independent of the luck distribution's hazard rate. 
The assumption of an increasing hazard rate is a standard one in the adverse-selection literature. ${ }^{3}$ In that work, the monotone-hazard-rate property refers to the distribution of players' types, whereas in our setting this property refers to the distribution of exogenous luck. However, all our results will completely remain the same if we reinterpret the random luck variable as the individual ability of a worker, characterizing his type and being unknown to each player ex ante. This reinterpretation only translates the standard moral-hazard setting into a moral-hazard model with symmetric ability uncertainty (see, e.g., Holmstrom 1999). Furthermore, irrespective of whether the random variable denotes luck or unknown ability in our model, the hazard rate technically works very similar compared to the adverse-selection models as it represents the trade-off between inducing incentives and the magnitude of expected payment (see Poblete and Spulber 2012). To sum up, accepting the monotone-hazard-rate property for the whole class of contract-theoretic models - including moral hazard - would imply that a bonus-pool contract outperforms individual bonus contracts under the assumption of limited liability.

Our paper is organized as follows. The next section summarizes the related literature. Section 3 introduces the model. Section 4 considers a specific example to illustrate our main finding on the optimality of bonus pools over individual bonuses. In Section 5, we derive the optimal bonuspool contract. Section 6 compares optimal individual bonus contracts with the optimal bonus-pool contract. Section 7 discusses the robustness of our main findings. Section 8 concludes.

\section{Related Literature}

Our paper is related to the previous literature on individual bonuses and bonus pools. Demougin and Fluet (1998), Wolfstetter (1999, pp. 288-294),

\footnotetext{
${ }^{3}$ See, e.g., the textbooks by Tirole (1988), p. 156, Wolfstetter (1999), p. 216, Bolton and Dewatripont (2005), p. 87, and Hermalin (2005), p. 102.
} 
and Oyer (2000) investigate the optimal contract in a second-best setting. They show that the optimal contract under risk neutral workers with limited liability is an individual bonus contract that attaches a fixed payment to a certain quota. Demougin and Fluet consider a setting with discrete outcomes and assume that the monotone likelihood ratio property holds. As a consequence, a bonus contract becomes optimal where the agent only receives a positive payment under the most favorable outcome. Similar to Oyer (2000), we assume outcome to be continuous. Of course, a contract that pays a positive bonus only under the most favorable outcome would now get into existence and characterization problems since the probability of the most favorable outcome is zero. We introduce two alternatives to get rid of this problem. First, we assume that the quota is bounded above. Alternatively, we follow the idea of Innes (1990) and assume that the principal is wealthconstrained. While the first alternative prevents tying a bonus to extremely high outcomes, the second alternative limits the magnitude of the optimal bonus payment.

Bonus-pool contracts have been discussed in the literature on the traditional personnel policy of Japanese firms and in the managerial-accounting literature. Kanemoto and MacLeod $(1989,1992)$ show that bonus-pool contracts solve the problem of subjective performance evaluation of Japanese firms. Baiman and Rajan (1995) also emphasize that bonus-pool contracts enable firms to make use of subjective performance evaluation. Rajan and Reichelstein (2006) and Budde (2007) analyze the optimal design of bonuspool contracts under subjective (and additional objective) performance measures. Contrary to these papers, we assume that output is described by a continuous variable, which conveys cardinal information on worker performance. Moreover, we solve for the optimal effort level and show that the optimal individual bonus contract and the optimal bonus-pool contract indeed lead to different efforts. All the previous papers on bonus pools do not directly contrast individual bonus contracts with a bonus-pool contract.

Beside theoretical contributions, the managerial-accounting literature also 
offers empirical work on bonus pools (e.g., Healy 1985; Gaver et al. 1995; Holthausen et al. 1995; Guidry et al. 1999). These studies mainly focus on how managers select accounting procedures to influence the magnitude of the bonus pools. Murphy and Oyer (2001) use data on management compensation to analyze the role of discretion in the distribution of bonus pools. Bonus pools are not only used to incentivize managers. From 2007 to 2010, New York City offered its teachers a bonus pool of $\$ 57$ million as incentive pay in addition to standard wages (Martinez 2011). Goodman and Turner (2013) empirically analyze the incentive effects of this bonus pool. They find that the bonus pool does not have a large impact on student achievement. The lack of success can be explained by the fact that the bonus pool was distributed among the schools according to performance, but within each school all teachers equally shared the performance pay, thus leading to possible free riding. All the examples mentioned in this paragraph show that the respective employers were able to use individual bonus contracts but they preferred bonus pools. The studies do not explain this preference. The advantage of bonus pools highlighted in our paper may be one reason, but there also exist further benefits of bonus pools which may be decisive as well (e.g., the commitment to an upper bound on overall labor costs).

Since the bonus-pool contract uses relative performance, our paper is also related to the literature on tournaments (e.g., Lazear and Rosen 1981; Green and Stokey 1983; Nalebuff and Stiglitz 1983). In particular, there are strong parallels to tournaments with a gap (Nalebuff and Stiglitz 1983, pp. 30-32; Eden 2007; Imhof and Kräkel 2011), because the bonus-pool contract also uses a gap or minimum distance to fine-tune incentives and the workers' probability of obtaining the high payment. The gap is somewhat related to handicaps, which are already addressed by the seminal paper of Lazear and Rosen (1981). Handicaps are imposed on stronger players to balance competition and, hence, to improve incentives. A gap, however, has to be beaten by all contestants. Moreover, the gap decreases incentives under an increasing hazard rate, but is nevertheless preferred by the employer to reduce 
worker rents.

Our paper is not the first one showing that an employer may prefer an incentive scheme that appears suboptimal according to Holmstrom's informativeness principle. Itoh (2004), Goel and Thakor (2006), Englmaier and Wambach (2010) and Bartling (2011) analyze incentive schemes in a situation where workers have other regarding preferences. Since inequity averse workers have to be compensated for their inequity costs under a binding participation constraint, it can be beneficial for the employer to base incentives on a less informative performance measure if the resulting incentive scheme leads to lower inequity costs. In particular, Englmaier and Wambach (2010) point out that an employer may prefer team incentives although they violate the informativeness principle since workers' tasks are unrelated. Paying team bonuses to workers is optimal because they result into less unequal payments. Weinschenk (2009, chapter IV) and Lang (2009) combine a principal-agent setting with ambiguity aversion and show that the optimal contract can lead to a violation of the informativeness principle. If the agent's ambiguity aversion is large enough, it will be optimal for the principal not to use an informative signal for creating incentives. Hence, the optimal contract boils down to a constant wage.

To the best of our knowledge, there only exists one paper that also compares individual incentive contracts with a bonus-pool contract, namely Koch and Peyrache (2011). Koch and Peyrache consider a two-period model with binary efforts, binary outputs and binary agent types. All players are risk neutral and agents are protected by limited liability. In this setting, it can be optimal for a principal to conceal information on agents' types to outsiders. For that purpose, the principal designs an opaque organization that makes agents' performance unobservable to the labor market so that an initially objective performance measure becomes subjective. Such policy leads to the following trade-off: on the one hand, uncertainty about agents' types creates career-concerns incentives (see Holmstrom 1999), which is beneficial for the principal. On the other hand, unverifiability of individual performance 
renders individual bonus contracts impossible. However, the principal can rely on the self-commitment property of a bonus pool to create incentives. Under certain conditions, the principal prefers an opaque organization that leads to a combination of career-concerns incentives and a bonus pool to a transparent organization which allows for the use of individual bonus contracts but eliminates career concerns. In our paper, we study under which conditions a bonus-pool contract dominates individual bonus contracts even in the absence of additional career-concerns incentives.

\section{The Model}

We consider a situation where an employer must hire two workers in order to run a firm. The three players are risk neutral. We assume that worker $i(i=1,2)$ chooses non-negative effort $e_{i}$ to increase the employer's profits. The employer observes the verifiable or objective performance measure ${ }^{4}$

$$
x_{i}\left(e_{i}\right)=e_{i}+\theta_{i}
$$

with $\theta_{1}$ and $\theta_{2}$ as random variables, denoting either noise or luck. The variables $\theta_{1}$ and $\theta_{2}$ are independent and identically distributed (i.i.d.) with density $f$ and cdf $F$, satisfying $\lim _{|\theta| \rightarrow \infty} f(\theta)=0$. We assume that $\int_{-\infty}^{\infty} f^{2}(\theta) d \theta<$ $\infty$ to guarantee that $\theta_{1}-\theta_{2}$ has a continuous density $g$ with corresponding cdf $G$. Both technical assumptions on $f$ are satisfied by all well-known densities. The probability distributions are common knowledge. The employer can neither observe $e_{i}$ nor $\theta_{i}$ so that we have a typical moral-hazard problem.

Exerting effort $e_{i}$ entails costs $c\left(e_{i}\right)$ for worker $i$ with $c(0)=c^{\prime}(0)=0$ and $c^{\prime}\left(e_{i}\right), c^{\prime \prime}\left(e_{i}\right), c^{\prime \prime \prime}\left(e_{i}\right)>0$ for $e_{i}>0 .{ }^{5}$ Let the workers' reservation values be

\footnotetext{
${ }^{4}$ See, e.g., Hermalin (2005), 155-156, on such a setting with an additively separable performance measure $x_{i}\left(e_{i}\right)$. In the main part of the paper, we follow this state-space formulation; see Hart and Holmstrom (1987), 77-78, and the cited literature on this approach. In the online appendix, we switch to the parameterized distribution formulation (Hart and Holmstrom 1987, 78) and show that our qualitative finding still holds.

${ }^{5}$ The assumption $c^{\prime \prime \prime}\left(e_{i}\right)>0$ is often used in moral-hazard models with limited liability
} 
$\bar{u}=0$. Workers are financially constrained so that their wage payments must be non-negative. ${ }^{6}$ Each worker maximizes expected net income, consisting of expected wage payment minus effort costs, whereas the employer maximizes $e_{1}+e_{2}$ minus expected wage payments.

In the following, we will compare two different contracts that are frequently used in practice. On the one hand, the employer can offer an individual bonus contract $\left(\hat{b}_{H i}, \hat{b}_{L i}, \hat{\gamma}_{i}\right)$ to each worker $i(i=1,2)$. Worker $i$ will receive the high bonus $\hat{b}_{H i}$ if his performance measure $x_{i}\left(e_{i}\right)$ exceeds a certain quota $\hat{\gamma}_{i}$; otherwise worker $i$ gets the low bonus $\hat{b}_{L i}\left(<\hat{b}_{H i}\right)$. On the other hand, the employer can fix a bonus pool $b_{H}+b_{L}$ and offer both workers a joint bonus-pool contract $\left(b_{H}, b_{L}, \gamma\right)$ based on relative performance. In analogy to the individual bonus contract, the variable $\gamma \geq 0$ denotes a threshold or gap by which worker $i$ must outperform worker $j$ in order to get the high bonus $b_{H}$. In other words, worker $i$ will only receive $b_{H}$ if $x_{i}\left(e_{i}\right)>x_{j}\left(e_{j}\right)+\gamma$. In that case, the inferior worker $j$ obtains the low bonus $b_{L}\left(<b_{H}\right)$. Like $\hat{\gamma}_{i}$ in the individual bonus contract, $\gamma$ is used to fine-tune incentives in the bonus-pool contract. As mentioned before, workers are protected by limited liability, implying $\hat{b}_{H i}, \hat{b}_{L i}, b_{H}, b_{L} \geq 0$. Recall that we assume noise to be i.i.d.. If $\theta_{1}$ and $\theta_{2}$ were not independent, then bonus pools would be desirable because they eliminate common noise (Holmstrom 1982, Green and Stokey 1983). Our story for why bonus pools may be desirable is completely different. We assume $\theta_{1}$ and $\theta_{2}$ are independent to focus the model on our main point.

To avoid the technical problems mentioned in Section 2, we consider two alternative restrictions. As a first alternative, we impose the restriction that the employer can only choose finite thresholds $\hat{\gamma}_{i}, \gamma \in[0, \bar{\gamma}]$ with $\bar{\gamma}>0$ and that $f$ and $g$ are strictly positive on $[0, \bar{\gamma}+\varepsilon]$ for some $\varepsilon>0$. In Germany, for example, industry-wide unions negotiate collective agreements with the employers' association concerning general conditions for employment conto guarantee strict concavity of the employer's objective function; see, e.g., Schmitz (2005), 730 .

${ }^{6}$ Alternatively, we can assume that legal minimum wages prohibit negative wages. 
tracts. We can imagine that such collective agreements restrict thresholds to a certain upper bound $\bar{\gamma}>0$. As a second alternative, we follow the suggestion of Innes (1990) and assume that the employer is wealth-constrained and possesses only capital $K>0$ when offering the workers a contract.

The timeline is the usual one in moral-hazard models: First, the employer offers the workers either individual bonus contracts $\left(\hat{b}_{H i}, \hat{b}_{L i}, \hat{\gamma}_{i}\right)$ or a bonuspool contract $\left(b_{H}, b_{L}, \gamma\right)$. Then, the workers can accept or reject the contract offer. If the workers accept, they will choose non-negative effort levels. Next, the random variables $\theta_{1}$ and $\theta_{2}$ are realized. Finally, the employer and the workers receive their payoffs according to the contract.

\section{An Illustrative Example}

In this example, we assume each $\theta_{i}$ is normally distributed with mean zero and variance $\sigma^{2}$, and that the employer seeks to implement a certain effort level.

The analysis in the following sections will show that the employer optimally chooses $\hat{b}_{L i}=b_{L}=0$, since workers have zero reservation values and are protected by limited liability. Under individual bonus contracts, worker $i$ exerts optimal effort that equates his marginal effort costs and marginal expected wage payments. Thus, the incentive constraint for individual bonus contracts can be written as

$$
c^{\prime}\left(e_{i}\right)=\hat{b}_{H i} \cdot f\left(\hat{\gamma}_{i}-e_{i}\right) .
$$

Under a bonus-pool contract, again worker $i$ 's optimal effort equates marginal effort costs and marginal expected wage payments. However, now wage payments depend on relative performance and, hence, the corresponding density $g$, which is again normal but has doubled variance (see, e.g., Wolfstetter 1999, 306). In a symmetric equilibrium, the two homogeneous workers choose identical efforts so that the incentive constraint under the bonus-pool contract reads as

$$
c^{\prime}\left(e_{i}\right)=b_{H} \cdot g(\gamma)
$$


The comparison of the incentive constraints (2) and (3) shows why, in agreement with Holmstrom's informativeness principle, individual bonuses seem to be better suited than a bonus pool to motivate workers. To implement a certain effort level at minimum costs, the employer chooses $\hat{\gamma}_{i}=e_{i}$ in (2) and $\gamma=0$ in (3). Hence, the bonus pool boils down to a simple tournament in which the worker with the higher performance receives $b_{H}$ as winner prize. Inserting $\hat{\gamma}_{i}=e_{i}$ and $\gamma=0$ leads to

$$
c^{\prime}\left(e_{i}\right)=\hat{b}_{H i} \cdot f(0) \quad \text { and } \quad c^{\prime}\left(e_{i}\right)=b_{H} \cdot g(0)
$$

with $f(0)=1 / \sqrt{2 \pi \sigma^{2}}$ and $g(0)=1 / \sqrt{4 \pi \sigma^{2}}$ and, thus, $f(0)>g(0)$. Intuitively, the relative performance measure $x_{i}\left(e_{i}\right)-x_{j}\left(e_{j}\right)$ is less precise than the performance measure $x_{i}\left(e_{i}\right)$ due to the doubled variance so that the density $g$ is flatter than $f$. As a consequence, for the same bonus payment $\hat{b}_{H i}=b_{H}$, marginal incentives are always lower under a bonus pool compared to individual bonuses since the impact of individual effort on performance outcome is less strong under relative performance evaluation due to a larger impact of noise.

Recall that the employer wants each worker to exert a certain effort level. Now suppose that the employer is wealth-constrained and possesses limited capital $K$. Thus, the highest possible effort that can be induced under individual bonuses, $\hat{e}^{*}$, is implicitly described by

$$
c^{\prime}\left(\hat{e}^{*}\right)=\frac{K}{2} \cdot f(0),
$$

whereas a bonus pool leads to maximum effort $e^{*}$ of each worker being described by

$$
c^{\prime}\left(e^{*}\right)=K \cdot g(0)
$$

Since $\frac{K}{2} \cdot f(0)<K \cdot g(0)$, for any fixed $K$, the maximum effort that can be implemented via individual bonuses is less than that which can be implemented by a bonus pool. As the example illustrates, bonus pools only use one collective payment to incentivize multiple workers which can lead to a crucial advantage under the assumption of a wealth-constrained employer 
that dominates the disadvantage suggested by Holmstrom's informativeness principle.

\section{The Optimal Bonus-Pool Contract}

In the following, the game is solved by backwards induction: First, we consider the workers' effort choices for a given bonus-pool contract $\left(b_{H}, b_{L}, \gamma\right)$ under the assumption that both workers participate. Then, we derive the optimal contract $\left(b_{H}^{*}, b_{L}^{*}, \gamma^{*}\right)$ that satisfies the workers' participation constraints, the incentive constraints and the limited-liability constraints.

We show in the appendix that the employer optimally withholds the complete bonus pool in case of a tie, i.e., if $\left|x_{1}\left(e_{1}\right)-x_{2}\left(e_{2}\right)\right| \leq \gamma$, and that in equilibrium the workers choose efforts $e_{1}=e_{2}=: e$ implicitly described by

$$
c^{\prime}(e)=\left(b_{H}-b_{L}\right) g(\gamma) .
$$

Equation (4) shows that, in equilibrium, each worker exerts the effort that equates marginal costs and the expected marginal gain from winning the relative performance evaluation. Intuitively, since each one gets at least $b_{L}$ irrespective of whether he wins, only the additional bonus payment $b_{H}-b_{L}$ creates incentives.

At the first stage of the game, the employer chooses the optimal bonuspool contract $\left(b_{H}^{*}, b_{L}^{*}, \gamma^{*}\right)$ that maximizes ${ }^{7}$

$$
2 e-2[1-G(\gamma)] \cdot\left(b_{L}+b_{H}\right)
$$

subject to the incentive constraint (4), the participation constraint $E U_{i}(e)$ $\geq 0$ and the limited-liability constraint $b_{L}, b_{H} \geq 0$. To solve for the optimal bonus-pool contract, we use the fact that the participation constraint is always satisfied: Each worker can ensure himself a non-negative expected utility - and, thus, at least his reservation value - by accepting any contract with non-negative payments and choosing zero effort. As a direct consequence, the

\footnotetext{
${ }^{7}$ We have $P\left(\left|x_{1}\left(e_{1}\right)-x_{2}\left(e_{2}\right)\right|>\gamma\right)=2[1-G(\gamma)]$.
} 
employer optimally chooses $b_{L}^{*}=0$ since positive payments would increase his expected labor costs and decrease workers' incentives (see (4)).

The employer thus solves

$$
\max _{e, \gamma, b_{H}} 2 e-2[1-G(\gamma)] b_{H} \stackrel{(4)}{=} \max _{e, \gamma} 2 \cdot\left(e-\frac{1-G(\gamma)}{g(\gamma)} c^{\prime}(e)\right) .
$$

According to (5), the employer faces the following trade-off when choosing the optimal gap $\gamma$ : On the one hand, he should choose a very large $\gamma$ to reduce the probability of paying out $b_{H}$ (implementation-cost effect). On the other hand, (4) indicates that a very large gap may also reduce incentives (incentive effect). If, for example, the convolution $g$ is a normal density, $g(\gamma)$ will decrease from the mean to the tail.

Let $r:=g /[1-G]$ denote the hazard rate of the difference $\theta_{i}-\theta_{j}$, and $\left(e^{*}, \gamma^{*}\right)$ the solution to problem (5), i.e., the employer chooses the gap $\gamma^{*}$ and implements effort $e^{*}$. We obtain the following result: ${ }^{8}$

Proposition 1 The employer offers the bonus-pool contract $\left(b_{H}^{*}, b_{L}^{*}, \gamma^{*}\right)$ with $b_{L}^{*}=0$.

(a) Suppose $\gamma$ is bounded above by $\bar{\gamma}$. Then the employer chooses $\gamma^{*} \in$ $\arg \max _{\gamma \in[0, \bar{\gamma}]} r(\gamma)$. Log-concavity of $g$ implies optimality of $\gamma^{*}=\bar{\gamma}$. For each worker the employer implements effort $e^{*} \in \arg \max _{e} e-c^{\prime}(e) / r\left(\gamma^{*}\right)$ via the high bonus $b_{H}^{*}=c^{\prime}\left(e^{*}\right) / g\left(\gamma^{*}\right)$.

(b) Suppose $r$ is increasing and the employer has limited wealth $K>0$. Define $\check{e}>0$ by $c^{\prime}(\check{e})=K g(0)$. Then $e^{*}$ maximizes $e-c^{\prime}(e) / r(\gamma(e))$ over $(0, \check{e}]$ and $\gamma^{*}=\gamma\left(e^{*}\right)$, where $\gamma(e):=\max \left\{\gamma \geq 0: K g(\gamma)=c^{\prime}(e)\right\}$. The employer chooses the high bonus $b_{H}^{*}=c^{\prime}\left(e^{*}\right) / g\left(\gamma^{*}\right)=K$.

Problem (5) shows that the employer wants to maximize the value of the hazard rate to trade off the implementation-cost effect against the incentive effect. This observation immediately leads to the results of Proposition 1(a). The hazard rate $r$ can have various shapes. ${ }^{9}$ If it is monotonic we will obtain

\footnotetext{
${ }^{8}$ All proofs are relegated to the appendix.

${ }^{9}$ See, e.g., Glaser (1980).
} 
a clear-cut result: In case of a monotonically decreasing (increasing) hazard rate the employer forgoes a gap (chooses the maximum possible gap $\bar{\gamma}$ ).

We know that the convolution $g$ has a peak at zero, which is also its global maximum. ${ }^{10}$ If the convolution is single-peaked, the hazard rate $r=$ $g /[1-G]$ is monotonically increasing in the negative domain up to the peak at zero since the numerator is increasing and the denominator decreasing. To the right of the peak the hazard rate will be still increasing if the decreasing denominator dominates the decreasing numerator. Proposition 1(a) includes this case, in which the implementation-cost effect dominates the incentive effect. This result holds in particular for the class of log-concave densities $g$ (e.g., for the normal distribution). ${ }^{11}$ For these distributions, the employer prefers $\gamma^{*}=\bar{\gamma}$ to minimize the probability of paying out the high bonus, $2[1-G(\gamma)]$. Since $g(\gamma)$ - and, hence, workers' incentives - become smaller the larger the gap $\gamma$, the employer has to compensate for the incentive effect by choosing an appropriately large bonus $b_{H}$ (see the incentive constraint (4)). Recall from the beginning of this paragraph that $\gamma=0$ maximizes workers' incentives. In contrast, under a log-concave density the employer minimizes incentives by the optimal gap $\gamma^{*}$ in order to minimize expected implementation costs as well.

Proposition 1(b) also refers to the case of an increasing hazard rate, but now the employer's limited wealth $K$ restricts the set of feasible $(e, \gamma)$ combinations when solving (5). Technically, the condition $\lim _{|\theta| \rightarrow \infty} f(\theta)=0$, which is satisfied by all well-known densities, together with the incentive constraint (4) makes the employer's limited-liability condition binding at the optimum (see (13) in the appendix):

$$
c^{\prime}(e)=K \cdot g(\gamma)
$$

The solution to the employer's problem now has two steps. First, for implementing a certain effort level $e$ at lowest possible cost, the employer chooses the largest corresponding gap $\gamma=\gamma(e)$ that satisfies equation (6). Second,

\footnotetext{
${ }^{10}$ See Lemma 1 in the proof of Proposition 1(b).

${ }^{11}$ See Bagnoli and Bergstrom (2005), section 6, for further examples.
} 
among all feasible $(e, \gamma(e))$-combinations the employer chooses the combination that solves (5). Recall from part (a) of Proposition 1 that if the hazard rate is increasing and the gap bounded above, the employer wants to combine a very large gap with a very large bonus $b_{H}$. Part (b) shows that if the employer is wealth-constrained we will have a similar solution since for given effort $e$ the employer still prefers the highest feasible gap (i.e., a gap that satisfies (6)).

\section{Comparison with Individual Bonus Con- tracts}

In the following, we will show that (1) although workers' tasks are completely unrelated and (2) although a bonus-pool contract in combination with relative performance evaluation leads to a less precise measure, the optimal bonus-pool contract can nevertheless dominate optimal individual bonus contracts.

The optimal bonus contract for each single worker $i,\left(\hat{b}_{H}^{*}, \hat{b}_{L}^{*}, \hat{\gamma}^{*}\right),{ }^{12}$ can be derived in two steps. First, workers' effort choice is analyzed for a given contract $\left(\hat{b}_{H}, \hat{b}_{L}, \hat{\gamma}\right)$. Second, the employer anticipates worker behavior and chooses the optimal contract that maximizes his expected net profits. We start with the step where each worker $i(i=1,2)$ maximizes his expected utility

$$
\hat{b}_{L}+\Delta \hat{b} \cdot P\left(x_{i}\left(e_{i}\right) \geq \hat{\gamma}\right)-c\left(e_{i}\right)=\hat{b}_{L}+\Delta \hat{b} \cdot\left[1-F\left(\hat{\gamma}-e_{i}\right)\right]-c\left(e_{i}\right)
$$

with $\Delta \hat{b}:=\hat{b}_{H}-\hat{b}_{L}$ denoting the bonus spread, and $F$ as cdf and $f$ as density of the i.i.d. random variables $\theta_{1}$ and $\theta_{2}$. As Oyer (2000) we assume that the worker's objective function is well-behaved and that optimal effort choice can

\footnotetext{
${ }^{12}$ Workers are homogeneous and do not interact. The employer, therefore, prefers the same optimal effort for each worker and chooses identical contracts. Hence, we suppress the subscript " $i$ " to simplify notation.
} 
be described by the first-order condition

$$
\Delta \hat{b} \cdot f\left(\hat{\gamma}-e_{i}\right)-c^{\prime}\left(e_{i}\right)=0 \Leftrightarrow \Delta \hat{b}=\frac{c^{\prime}\left(e_{i}\right)}{f\left(\hat{\gamma}-e_{i}\right)} .
$$

In stage 1, the employer optimally designs the individual bonus contract. In analogy to the optimal bonus-pool contract, the workers' limited-liability constraint implies the participation constraint as $\bar{u}=c(0)=0$, and the employer chooses $\hat{b}_{L}^{*}=0$ to minimize his labor costs. Thus, for each worker the employer solves

$$
\begin{aligned}
& \max _{\Delta \hat{b}, \hat{\gamma}, e_{i}} e_{i}-\hat{b}_{H} \cdot P\left(x_{i}\left(e_{i}\right) \geq \hat{\gamma}\right) \quad \text { s.t. (7) } \\
& =\max _{\hat{\gamma}, e_{i}} e_{i}-\frac{1-F\left(\hat{\gamma}-e_{i}\right)}{f\left(\hat{\gamma}-e_{i}\right)} c^{\prime}\left(e_{i}\right)
\end{aligned}
$$

Problems (5) and (8) look similar because in each case the employer wants to maximize a hazard rate: for the bonus-pool contract the hazard rate $r=g /(1-G)$ of $\theta_{1}-\theta_{2}$, and for the individual bonus contract the hazard rate $r_{\theta}=f /(1-F)$ of $\theta_{i}$. For an intuitive explanation of why the employer seeks to maximize the hazard rate consider worker $i$ evaluating effort level $e_{i}$ under an individual bonus contract $\left(\hat{b}_{H}, \hat{b}_{L}, \hat{\gamma}\right)$. To obtain the high bonus, the worker must have some amount of luck: $\theta_{i} \geq \hat{\gamma}-e_{i}$. Assuming he has the required amount of luck, the worker is interested in the conditional probability that his performance will exceed the threshold significantly, say, $x_{i}\left(e_{i}\right)>\hat{\gamma}+\delta$ with some small $\delta>0$. The probability is related to the hazard rate by $P\left(x_{i}\left(e_{i}\right)>\hat{\gamma}+\delta \mid \theta_{i} \geq \hat{\gamma}-e_{i}\right) \approx 1-\delta r_{\theta}\left(\hat{\gamma}-e_{i}\right)$. The larger this probability, the weaker the incentive for the worker to increase his effort. This effect explains why a large value of $r_{\theta}\left(\hat{\gamma}-e_{i}\right)$ is in the interest of the employer. A similar argument applies in the case of a bonus-pool contract.

A comparison of individual bonus contracts and bonus-pool contracts leads, in view of (5) and (8), to a comparison of the hazard rates $r_{\theta}$ and $r$. Given that the thresholds of both contract types are equally constrained (i.e., $\gamma, \hat{\gamma} \in[0, \bar{\gamma}]$ ), the optimal bonus-pool contract will dominate individual contracts if $r(\alpha) \geq r_{\theta}(\alpha)$ for all $\alpha \geq 0$, that is, if $\theta_{1}-\theta_{2}$ is smaller than $\theta_{1}$ in 
the hazard rate order, see e.g. Shaked and Shanthikumar (2007). A sufficient condition for this to be the case is that $\theta_{i}$ has an increasing hazard rate.

Proposition 2 Suppose the density $f$ is log-concave or, more generally, the hazard rate $r_{\theta}$ of $\theta_{i}$ is increasing.

(a) Suppose $\theta_{1}$ and $\theta_{2}$ are non-negative and the thresholds are bounded above (i.e., $\gamma, \hat{\gamma} \in[0, \bar{\gamma}]$ ). Then the optimal bonus-pool contract will dominate all individual bonus contracts. If $r_{\theta}$ is strictly increasing, using the optimal bonus pool instead of two bonus contracts will lead to a strict improvement.

(b) Suppose the employer has limited wealth $K>0$. Suppose further that the hazard rate $r$ of $\theta_{1}-\theta_{2}$ is increasing as well and that effort $\bar{e}>0$ is implementable by either contract. Let

$$
\begin{gathered}
\gamma(\bar{e}):=\max \left\{\gamma \geq 0: K g(\gamma)=c^{\prime}(\bar{e})\right\} \\
\text { and } \quad \hat{\gamma}(\bar{e}):=\max \left\{\hat{\gamma} \geq 0: \frac{K}{2} f(\hat{\gamma}-\bar{e})=c^{\prime}(\bar{e})\right\} .
\end{gathered}
$$

Then the bonus-pool contract implementing $\bar{e}$ at minimal costs sets $\gamma(\bar{e})$, the individual bonus contract implementing $\bar{e}$ at minimal costs sets $\hat{\gamma}(\bar{e})$, and the bonus pool is less costly if and only if $r(\gamma(\bar{e}))>r_{\theta}(\hat{\gamma}(\bar{e})-\bar{e})$.

Proposition 2(a) deals with the scenario considered by Kim (1997), Oyer (2000), Poblete and Spulber (2012) and others who assume that the $\theta_{i}$ can only take non-negative values. The authors motivate their setting by assuming that the performance measure $x_{i}$ describes physical output or realized sales so that the exclusion of negative realizations seems reasonable. Part (a) points out that for a wide class of probability distributions, including truncated normal and uniform distributions, the optimal bonus contract can be strictly improved by combining workers' incentives via a bonus-pool contract. Whereas this result holds for exogenously constrained thresholds, Proposition 2(b) refers to endogenous thresholds and does not restrict the possible realizations of the $\theta_{i}$. According to (b), a finding similar to part (a) will hold for thresholds that are endogenously constrained by the employer's limited wealth if the optimal thresholds for the two contract types do not differ too 
much. Then, the hazard-rate-order result of part (a) (i.e., $r(\alpha)>r_{\theta}(\alpha)$ for all $\alpha \geq 0$, see the proof in the appendix) is still decisive for the comparison between the bonus pool and individual bonus contracts and the employer can implement a certain effort level at lower expected costs by using a bonus pool instead of two individual bonus contracts.

Against the background of Holmstrom's informativeness principle, the results of Proposition 2 seem surprising as the workers' tasks are neither technically nor stochastically related. ${ }^{13}$ A bonus-pool contract uses relative performance evaluation, and the relative performance measure $x_{1}\left(e_{1}\right)-x_{2}\left(e_{2}\right)$ is less precise than the measure $x_{i}\left(e_{i}\right)$ used by an individual bonus contract. ${ }^{14}$ Correspondingly, it is plausible that the implementation of a given effort level requires a bonus pool, $b_{H}$, that is larger than the total bonus, $2 \hat{b}_{H}$, offered under two individual contracts.

However, for implementing a certain effort level $e$, it is not the size of the bonus, but the expected costs, that the employer is primarily interested in. The probability that the bonus pool is paid out is $2[1-G(\gamma)]$ and the probability that the individual bonus is paid out is $1-F(\hat{\gamma}-e)$. The expected costs under the bonus-pool contract and under the individual contracts are therefore $2 b_{H}[1-G(\gamma)]$ and $2 \hat{b}_{H}[1-F(\hat{\gamma}-e)]$. As argued above, given effort $e$, the corresponding bonus pool $b_{H}$ should be larger than total bonus $2 \hat{b}_{H}$. However, the implementation-cost effect mentioned in Section 5, which focuses on the probability of paying out $b_{H}$ or $2 \hat{b}_{H}$, respectively, works into the opposite direction. Especially, if the $\theta_{i}$ are non-negative, $1-G(\gamma)<$ $1-F(\gamma) \leq 1-F(\gamma-e)$. Hence, the probability of paying out the bonus pool tends to be smaller than the probability of paying the high bonuses to both workers under individual contracts. Proposition 2 provides conditions under which the implementation-cost effect is strong enough to dominate the incentive downside so that the employer prefers a bonus pool to individual

\footnotetext{
${ }^{13}$ See Holmstrom (1982) and Mookherjee (1984) for the multi-agent case.

${ }^{14} \operatorname{Var}\left(\theta_{1}-\theta_{2}\right)=2 \operatorname{Var}\left(\theta_{i}\right)$, so that the relative performance measure is half as precise as the absolute performance measure for a single worker, $x_{i}\left(e_{i}\right)$.
} 
bonuses. ${ }^{15}$ Since workers are protected by limited liability and earn positive rents, a dominance of the bonus-pool contract over individual bonus contracts implies that the employer benefits from reduced worker rents when pooling incentives.

Less technically, the advantage of a bonus pool in connection with relative performance evaluation can be explained as follows. The previous results have shown that, due to the increasing hazard rate, the employer wants to combine a large threshold with large bonus payments under both individual bonuses and a bonus pool. The latter one has two advantages in this situation. First, if the quota for individual bonuses is exogenously constrained to $\hat{\gamma}=\bar{\gamma}$, the employer will strictly benefit from using $\bar{\gamma}$ as minimum distance and letting the workers compete against each other via relative performance evaluation. Competition implies that each worker now has to beat $\bar{\gamma}$ and his opponent which leads to an extension of the original threshold and, thereby, a reduction of worker rents via the implementation-cost effect. Second, under a bonus pool several workers compete for only one large bonus payment, whereas under individual bonuses each worker has to be incentivized by a separate wage premium. If the employer is wealth-constrained, one big bonus pool may work better than several individual bonuses, which are rather small since the employer is financially constrained.

In Proposition 2 we did not address the possibility that the employer may be unable to implement certain effort levels under one of the contracts. However, if the employer is wealth-constrained it may be the case that combining a large threshold with a high bonus payment is not feasible since the employer's amount of liability is too small. Considering the implementability of effort under the two types of contracts leads to the following results, which do not impose restrictions on the hazard rates:

Proposition 3 Suppose the employer has limited wealth $K>0$.

\footnotetext{
${ }^{15}$ An example with a specific distribution can be used to illustrate the previous arguments and the magnitude by which the bonus-pool contract may outperform the bonus contract; see the online appendix.
} 
(a) Let $\theta_{1}, \theta_{2} \in[0, \infty)$. There exists $e_{0} \in[0, \infty]$, which depends only on $f$ and $K$, but not on $c$, such that the following holds: (a1) Every nonnegative effort level $e<e_{0}$ that can be implemented by individual bonus contracts can also be implemented by a bonus-pool contract. (a2) Every finite effort level $e>e_{0}$ that can be implemented by a bonus-pool contract can also be implemented by individual bonus contracts.

(b) If an effort level e satisfies

$$
\max _{\hat{\gamma}} f(\hat{\gamma}-e) \frac{K}{2}<c^{\prime}(e) \leq g(0) K,
$$

this effort will be implementable by a bonus pool but not by individual bonus contracts.

According to Proposition 3(a), there exists a critical effort level, $e_{0}$, so that some smaller (larger) effort levels may be implementable by a bonus pool (individual bonuses) but not by individual bonuses (a bonus pool). ${ }^{16}$ Part (b) of Proposition 3 does not restrict the possible realizations of the $\theta_{i}$. It shows how the employer's limited wealth, $K$, favors the implementability of effort by a bonus pool compared to individual bonuses and gives a sufficient condition for an effort level to be only implementable by a bonus pool.

The intuition for our findings is the following. The incentive constraints (4) and (7) show that, under either contract, both the bonus payment in case of success (i.e., $b_{H}$ and $\hat{b}_{H}$, respectively) and the marginal winning probability (i.e., $g(\gamma)$ and $f(\hat{\gamma}-e)$, respectively) have to be sufficiently large for the implementation of a certain effort level. The bonus payment in case of success is restricted by the employer's limited-liability constraint, which depends on $K$ under a bonus pool and on $K / 2$ under individual bonuses. Since the amount of liability per worker is twice as high under a bonus pool than under individual bonuses, the employer may implement certain efforts solely via the bonus pool if this liability advantage is not outweighed by a sufficiently larger marginal winning probability. ${ }^{17}$

\footnotetext{
${ }^{16}$ An illustrating example can be requested from the authors.

${ }^{17} g(0)=\max _{\delta} g(\delta)<\max _{\theta} f(\theta)$ since under the convolution the total probability mass is now distributed over the doubled support.
} 
Part (b) of Proposition 3 captures the main idea of the example in Section 4 , which deals with normally distributed $\theta_{i}$, leading to $\max _{\hat{\gamma}} f(\hat{\gamma}-e) \frac{K}{2}<$ $g(0) K$. Thus, for small values of $K$, many efforts are not implementable by either contract. For larger values of $K$, there exists a range of effort levels that are implementable via a bonus pool but not via individual bonuses. If $K$ is sufficiently large, many moderate effort levels can be implemented by either using a bonus pool or individual bonuses.

To sum up, the results of Section 6 have shown that complementing individual bonus contracts by the possibility of pooling incentives may not only save rents for the employer but also increase the set of implementable effort levels.

\section{Discussion}

We have shown under which conditions a firm that employs two workers prefers a bonus pool to individual bonus contracts. This dominance of a bonus-pool contract will still hold if we extend our setting to the case of more than two workers. In that case, the employer can divide the set of workers into pairs of two workers whose incentives are combined by a bonuspool contract. If the number of workers, say $n$, is even, there will be $n / 2$ bonus pools; if $n$ is odd, the employer can design $(n-1) / 2$ bonus pools and one individual bonus contract. If pooling of more than two workers does not lead to worker discouragement when competing for the bonus payments, the outcome of optimal bonus-pool contracts can be even further improved.

In the paper, we often refer to the case of an increasing hazard rate. This assumption holds for many well-known distributions like the normal distribution and the uniform distribution. Less clear-cut results can be derived for non-monotonic hazard rates. However, not all findings are based on an increasing hazard rate. In particular, Proposition 3 on the implementability of given effort levels does not impose any restriction on the shape of the hazard rate. 
Condition (9) shows that a bonus pool can be less effective if the convolution $g$ becomes very flat compared to the initial density $f$. In that case, incentives are rather small under a bonus pool for given bonus payments. Hence, the bonus pool will only dominate individual bonus contracts if the comparative advantage of financing incentives for all workers together via $K$ exceeds the disadvantage of a small $g(0)$.

Throughout the paper we assumed that the performance measures $x_{i}\left(e_{i}\right)$ are verifiable. Skipping this assumption without adding supplementary assumptions like repeated interaction or third-party contracting would render the use of individual bonuses impossible whereas bonus pools still work due to their self-commitment property, which has been highlighted by Malcomson (1984).

Finally, one can ask whether our results are robust to the formulation of the moral-hazard problem used in this paper. As a robustness check, we can switch from the state-space formulation to the parameterized distribution formulation, where effort choice shifts probability mass over possible outcomes (e.g., Hart and Holmstrom 1987, 78). ${ }^{18}$ We can show that the advantage of pooling bonus payments in situations with a financially constrained employer also holds under this alternative modeling of our moral-hazard problem. ${ }^{19}$

Bonus contracts have the beneficial characteristic that the performance target or threshold can be used by the employer as an effective instrument to fine-tune incentives. Since the threshold does not only influence workers' incentives but also the probability that a bonus payment occurs - and, hence, the magnitude of worker rents - the employer has to trade off both effects when choosing the optimal threshold. In case of a bonus-pool contract, the employer faces a similar optimization problem. The central result of this paper has shown that - for a wide class of probability distributions - a bonus pool offers a better solution to the trade off than individual bonus contracts.

\footnotetext{
${ }^{18}$ We thank a referee for pointing to this alternative.

${ }^{19}$ See the online appendix for details.
} 


\section{Appendix}

Workers' equilibrium efforts under the optimal bonus-pool contract:

Suppose the employer uses a bonus pool in combination with relative-performance evaluation. That is, worker $i$ receives payment $w\left(x_{i}-x_{j}\right)$ with $w$ : $\mathbb{R} \rightarrow[0, \infty)$ being non-decreasing and $x_{k} \equiv x_{k}\left(e_{k}\right)(k=i, j)$ according to (1). For a given scheme $w\left(x_{1}-x_{2}\right)$, worker 1 maximizes

$$
\begin{aligned}
E U_{1}\left(e_{1}\right) & =E\left[w\left(x_{1}-x_{2}\right)\right]-c\left(e_{1}\right) \\
& =\int_{-\infty}^{\infty} w\left(e_{1}-e_{2}+t\right) g(t) d t-c\left(e_{1}\right)
\end{aligned}
$$

where $g$ denotes the density of $\theta_{1}-\theta_{2}$. Using integration by substitution and symmetry of $g$ (recall that the $\theta_{i}$ are i.i.d.) we obtain

$$
E U_{1}\left(e_{1}\right)=\int_{-\infty}^{\infty} w(v) g\left(e_{1}-e_{2}-v\right) d v-c\left(e_{1}\right)
$$

Similarly, worker 2's objective function reads as

$$
E U_{2}\left(e_{2}\right)=\int_{-\infty}^{\infty} w(v) g\left(e_{2}-e_{1}-v\right) d v-c\left(e_{2}\right)
$$

We assume that an equilibrium in pure strategies exists and is characterized by the first-order conditions ${ }^{20}$

$$
\begin{aligned}
\int_{-\infty}^{\infty} w(v) g^{\prime}\left(e_{1}-e_{2}-v\right) d v & =c^{\prime}\left(e_{1}\right) \\
\text { and } \quad \int_{-\infty}^{\infty} w(v) g^{\prime}\left(e_{2}-e_{1}-v\right) d v & =c^{\prime}\left(e_{2}\right) .
\end{aligned}
$$

Since workers are homogeneous, we concentrate on the characterization of symmetric equilibria, ${ }^{21}$ leading to $e_{1}=e_{2}$. Inserting the symmetry condition

\footnotetext{
${ }^{20}$ Pure-strategy equilibria will exist if the cost of effort function is sufficiently steep and the density $g$ sufficiently flat so that the workers' objective functions are concave in the relevant range; see, e.g., Lazear and Rosen (1981), p. 845, Nalebuff and Stiglitz (1983), p. 29. See Wolfstetter (1999, p. 305), Schöttner (2008) and Gürtler (2011) for sufficient conditions that guarantee existence. Of course, existence can be proved for given specifications of the noise distribution.

${ }^{21}$ Symmetric equilibria seem to be most plausible since workers are completely homogeneous. The symmetric equilibrium is unique. However, in our general setting we cannot exclude the existence of additional asymmetric equilibria.
} 
$e_{1}=e_{2}=: e$ into the first-order conditions yields ${ }^{22}$

$$
c^{\prime}(e)=-\int_{-\infty}^{\infty} w(v) g^{\prime}(v) d v .
$$

In the next step, we will show that the employer can at least weakly improve the relative-performance scheme $w$ by switching to the alternative scheme

$$
w^{*}(v)=\left\{\begin{array}{cc}
0 & \text { if } v \leq 0 \\
w(v)-w(-v) & \text { otherwise }
\end{array}\right.
$$

which is non-negative, non-decreasing and, moreover, satisfies $w^{*}(v) \leq w(v)$ for all $v$. Integration by substitution and the symmetry of $g$ yield

$$
\begin{aligned}
\int_{-\infty}^{\infty} w^{*}(v) g^{\prime}(v) d v=\int_{0}^{\infty} w(v) g^{\prime}(v) d v-\int_{0}^{\infty} w(-v) g^{\prime}(v) d v \\
=\int_{0}^{\infty} w(v) g^{\prime}(v) d v+\int_{0}^{-\infty} w(v) g^{\prime}(-v) d v \\
=\int_{0}^{\infty} w(v) g^{\prime}(v) d v+\int_{-\infty}^{0} w(v) g^{\prime}(v) d v=\int_{-\infty}^{\infty} w(v) g^{\prime}(v) d v
\end{aligned}
$$

Hence, from (10) and the fact that $w^{*}(v) \leq w(v)$ we see that replacing scheme $w$ by scheme $w^{*}$ leads to the implementation of the same effort level $e$ at weakly lower costs for the employer. This useful result sheds light on the optimal distribution of a bonus pool: the best an employer can do is paying zero to a worker that is weakly outperformed by his co-worker.

The result also gives clear advice to the employer how to behave in case of the bonus-pool contract $\left(b_{H}, b_{L}, \gamma\right)$ defined in Section 3 if a tie occurs, i.e., if $\left|x_{1}\left(e_{1}\right)-x_{2}\left(e_{2}\right)\right| \leq \gamma$. If the performance measure $x_{i}\left(e_{i}\right)$ is unverifiable, the employer has to pay out the full bonus pool even in case of a tie in order to meet Malcomson's (1984) self-commitment property; otherwise he would opportunistically claim that state of the world that leads to the lowest labor costs. Following the equal-sharing rule of Nalebuff and Stiglitz (1983), pp.

\footnotetext{
${ }^{22} g^{\prime}(-v)=-g^{\prime}(v)$ due to the symmetry of $g$.
} 
30-32, the payment to worker $i$ would read as

$$
w(v)=\left\{\begin{array}{ccc}
b_{L} & \text { if } & v<-\gamma \\
\frac{b_{L}+b_{H}}{2} & \text { if } & -\gamma \leq v \leq \gamma \\
b_{H} & \text { if } & v>\gamma
\end{array}\right.
$$

with $v=x_{i}-x_{j}$. However, in the given setting with measure $x_{i}\left(e_{i}\right)$ being verifiable, relative performance pay need not satisfy the self-commitment property. Instead, the employer should replace incentive scheme (12) by the alternative scheme (11) to implement equilibrium effort $e$ at lower costs. Thus, we obtain

$$
w^{*}(v)=\left\{\begin{array}{cc}
0 & \text { if } v \leq \gamma \\
b_{H}-b_{L} & \text { if } v>\gamma
\end{array}\right.
$$

and

$$
c^{\prime}(e)=-\int_{-\infty}^{\infty} w^{*}(v) g^{\prime}(v) d v=\left(b_{H}-b_{L}\right) g(\gamma)
$$

Proof of Proposition 1:

(a) Objective function (5) shows that the employer prefers the gap $\gamma^{*}$ that maximizes $r(\gamma)$ in order to minimize expected implementation costs for a certain effort level $e$. Then for given $\gamma^{*}$, the employer implements optimal effort $e^{*}$ that solves (5) by fine-tuning incentives via $b_{H}$ according to (4). If $r$ is monotonically increasing, then $\gamma^{*}=\bar{\gamma}$ is optimal. An (1998) and Bagnoli and Bergstrom (2005) show that log-concavity of a density function implies that this density has an increasing hazard rate, leading to $\gamma^{*}=\bar{\gamma}$.

(b) The proof uses the following auxiliary result:

Lemma 1 The global maximum of the convolution $g$ is attained at zero.

Proof Recall that $\theta_{1}$ and $\theta_{2}$ are i.i.d. with density $f$. In view of the assumption that $\theta_{1}-\theta_{2}$ has a continuous density $g, g(\gamma)=\int_{-\infty}^{\infty} f(\theta) f(\theta-\gamma) d \theta$ for every $\gamma \in \mathbb{R}$, see, e.g., Mood et al. (1974), pp. 185-186, for the convolution 
formula. Applying the Cauchy-Schwarz inequality leads to ${ }^{23}$

$$
\begin{aligned}
g(\gamma) & =\int_{-\infty}^{\infty} f(\theta) f(\theta-\gamma) d \theta \leq \sqrt{\int_{-\infty}^{\infty}[f(\theta)]^{2} d \theta} \sqrt{\int_{-\infty}^{\infty}[f(\theta-\gamma)]^{2} d \theta} \\
& =\int_{-\infty}^{\infty}[f(\theta)]^{2} d \theta=g(0) \quad \text { for all } \gamma \cdot \square
\end{aligned}
$$

If the employer has limited wealth $K$ he will face the additional constraint $b_{H} \leq K$, which, by (4), is equivalent to

$$
c^{\prime}(e) \leq K g(\gamma)
$$

Since $c^{\prime}$ is strictly increasing, there exists $\gamma \geq 0$ satisfying (13) if and only if $e \in[0, \check{e}]$ where $\check{e}$ with $c^{\prime}(\check{e})=K g(0)$ defines the maximally implementable effort level according to Lemma 1. Since $r(\gamma)$ is increasing and the employer has to solve (5), for any effort level $e \in(0, \check{e}]$ that he wants to implement he chooses, if possible, the largest corresponding $\gamma$ that satisfies (13). The condition $\lim _{|\theta| \rightarrow \infty} f(\theta)=0$ implies that $\lim _{\gamma \rightarrow \infty} g(\gamma)=0$ and, hence, that the right-hand side of (13) goes to zero as $\gamma \rightarrow \infty$. It follows that for every $e \in(0, \check{e}]$, there exists a largest $\gamma$ satisfying (13), and for this $\gamma,(13)$ must be binding, so that $\gamma$ coincides with $\gamma(e)$ as defined in Proposition 1(b). In view of (5), the optimal effort $e^{*}$ maximizes $e-c^{\prime}(e) / r(\gamma(e))$.

Proof of Proposition 2:

According to An (1998) and Bagnoli and Bergstrom (2005), $r_{\theta}$ will be monotonically increasing if $f$ is log-concave.

(a) We show strict improvement for the case of a strictly increasing hazard rate $r_{\theta}$; proving weak dominance for an increasing hazard rate proceeds analogously. According to (5) and (8), any effort level $e \in(0, \bar{\gamma}]$ can be implemented at a lower cost under a bonus-pool contract compared to an individual bonus contract if ${ }^{24}$

$$
\max _{0 \leq \gamma \leq \bar{\gamma}} \frac{g(\gamma)}{1-G(\gamma)}>\frac{f(\bar{\gamma}-e)}{1-F(\bar{\gamma}-e)} \Leftrightarrow \max _{0 \leq \gamma \leq \bar{\gamma}} r(\gamma)>r_{\theta}(\bar{\gamma}-e) .
$$

${ }^{23}$ The step from line 1 to line 2 uses the fact that $\int_{\underline{x}}^{\bar{x}} y(x-\alpha) d x=\int_{\underline{x}-\alpha}^{\bar{x}-\alpha} y(x) d x$.

24 (5) describes the employer's maximization problem for both workers. 
Let $\beta:=\sup \{x \in \mathbb{R}: F(x)<1\}$. Using the convolution formula $g(\alpha)=$ $\int_{0}^{\infty} f(\alpha+\theta) f(\theta) d \theta$ (e.g., Mood et al. 1974, p. 185) we obtain for all $\alpha \in$ $[0, \bar{\gamma})^{25}$

$$
\begin{gathered}
r(\alpha)=\frac{g(\alpha)}{1-G(\alpha)}=\frac{\int_{0}^{\infty} f(\alpha+\theta) f(\theta) d \theta}{1-\int_{0}^{\infty} F(\alpha+\theta) f(\theta) d \theta} \\
=\frac{\int_{0}^{\beta-\alpha} r_{\theta}(\alpha+\theta)[1-F(\alpha+\theta)] f(\theta) d \theta}{\int_{0}^{\beta-\alpha}[1-F(\alpha+\theta)] f(\theta) d \theta} \\
>\frac{\int_{0}^{\beta-\alpha} r_{\theta}(\alpha)[1-F(\alpha+\theta)] f(\theta) d \theta}{\int_{0}^{\beta-\alpha}[1-F(\alpha+\theta)] f(\theta) d \theta}=r_{\theta}(\alpha)
\end{gathered}
$$

where the inequality follows from the strict monotonicity of $r_{\theta}$. Thus, combining effort $e$ with the gap $\gamma=\bar{\gamma}-e$ under a bonus pool leads to strictly lower implementation costs compared to individual bonus contracts.

(b) It was shown in the proof of Proposition 1(b) that the bonus-pool contract implementing $\bar{e}$ at minimal costs has the gap $\gamma(\bar{e})$ as defined in Proposition 2(b). Under two individual bonus contracts, the employer can use wealth $K / 2$ for paying the bonus $\hat{b}_{H}$ to each worker. Combining the employer's limited-liability condition $K / 2 \geq \hat{b}_{H}$ and incentive constraint (7) yields that $c^{\prime}(\bar{e}) \leq f(\hat{\gamma}-\bar{e}) K / 2$ must hold for implementing $\bar{e}$ under individual bonus contracts. Since $r_{\theta}$ is increasing and $\lim _{|\theta| \rightarrow \infty} f(\theta)=0$, objective function (8) shows that the optimal quota for implementing $\bar{e}$ at lowest cost is given by $\hat{\gamma}(\bar{e})$ as defined in Proposition 2(b). In view of (5) and (8), the bonus pool has lower implementation costs if and only if $r(\gamma(\bar{e}))>r_{\theta}(\hat{\gamma}(\bar{e})-\bar{e})$.

Proof of Proposition 3:

(a) Define $\nu$ and $\mu(e)$ by

$$
\nu:=K g(0), \quad \mu(e):=\max _{\hat{\gamma} \geq e} \frac{K}{2} f(\hat{\gamma}-e) .
$$

It was shown in the proof of Proposition 1(b) that effort $e$ can be implemented by a bonus-pool contract if and only if $c^{\prime}(e) \leq \nu$. It was shown in the proof

\footnotetext{
${ }^{25}$ See Miravete (2005), p. 1358, on a similar proof for the sum of two random variables, $\xi_{i}+\xi_{j}$.
} 
of Proposition 2(b) that effort $e$ can be implemented by individual bonus contracts if and only if $c^{\prime}(e) \leq \mu(e)$. The function $\mu$ is increasing. To see this let $0 \leq e_{1}<e_{2}$ and let $\hat{\gamma}_{1} \geq e_{1}$ be such that $\frac{K}{2} f\left(\hat{\gamma}_{1}-e_{1}\right)=\mu\left(e_{1}\right)$. Let $\hat{\gamma}_{2}=\hat{\gamma}_{1}-e_{1}+e_{2}$. Then $\hat{\gamma}_{2} \geq e_{2}$ and since $e_{2}>e_{1}, \hat{\gamma}_{2}>\hat{\gamma}_{1}$. Therefore,

$$
\mu\left(e_{2}\right) \geq \frac{K}{2} f\left(\hat{\gamma}_{2}-e_{2}\right)=\frac{K}{2} f\left(\hat{\gamma}_{1}-e_{1}\right)=\mu\left(e_{1}\right) .
$$

It now follows that there exists $e_{0} \in[0, \infty]$ so that $\mu(e) \leq \nu$ if $0 \leq e<e_{0}$, and $\mu(e) \geq \nu$ if $e_{0}<e<\infty$. This proves (a1) and (a2).

(b) The claim directly follows from the considerations in the proofs of Propositions 1(b) and 2(b). The thresholds that solve both sides of condition (9) are finite due to $\lim _{|\theta| \rightarrow \infty} f(\theta)=0$.

\section{References}

An, M.Y., 1998. Logconcavity versus logconvexity: a complete characterization. Journal of Economic Theory 80, 350-369.

Bagnoli, M., Bergstrom, T., 2005. Log-concave probability and its applications. Economic Theory 26, 445-469.

Baiman, S., Rajan, M.V., 1995. The informational advantages of discretionary bonus schemes. Accounting Review 70, 557-579.

Bartling , B., 2011. Relative performance or team evaluation? Optimal contracts for other-regarding agents. Journal of Economic Behavior and Organization 79, 183-193.

Bolton, P., Dewatripont, M., 2005. Contract theory. MIT Press, Cambridge (Mass.).

Budde, J., 2007. Bonus pools, limited liability, and tournaments. SFB/TR 15 (Governance and the Efficiency of Economic Systems), Discussion Paper No. 205. 
Demougin, D., Fluet, C., 1998. Mechanism sufficient statistic in the riskneutral agency problem. Journal of Institutional and Theoretical Economics $154,622-639$.

Eden, M., 2007. Tournaments as optimal contracts. Mimeo.

Englmaier, F., Wambach, A., 2010. Optimal incentive contracts under inequity aversion. Games and Economic Behavior 69, 312-328.

Gaver,J.J., Gaver, K.M., Austin, J.R., 1995. Additional evidence on bonus plans and income management. Journal of Accounting and Economics $19,3-28$.

Glaser, R.E., 1980. Bathtub and related failure rate characterizations. Journal of the American Statistical Association 75, 667-672.

Goel, A.M., Thakor, A.V., 2006. Optimal contracts when agents envy each other. Mimeo.

Goodman, S.F., Turner, L.J., 2013. The design of teacher incentive pay and educational outcomes: Evidence from the New York City bonus program. Journal of Labor Economics 31, 409-420.

Green, J.R., Stokey, N.L., 1983. A comparison of tournaments and contracts. Journal of Political Economy 91, 349-364.

Gürtler, O., 2011. The first-order approach in rank-order tournaments. Economics Letters 111, 185-187.

Guidry, F., Leone, A.J., Rock, S., 1999. Earnings-based bonus plans and earnings management by business-unit managers. Journal of Accounting and Economics 26, 113-142.

Hart, O., Holmstrom, B., 1987. The theory of contracts. In: Bewley, T.F., Ed., Advances in economic theory. Fifth world congress. Cambridge University Press, New York et al. 
Healy, P.M., 1985. The effect of bonus schemes on accounting decisions. Journal of Accounting and Economics 7, 85-107.

Hermalin, B.E., 2005. Lecture notes for economics.

Herweg, F., Müller, D., Weinschenk, P., 2010. Binary payment schemes: moral hazard and loss aversion. American Economic Review 100, 24512477 .

Holmstrom, B., 1979. Moral hazard and observability. Bell Journal of Economics 10, 74-91.

Holmstrom, B., 1982. Moral hazard in teams. Bell Journal of Economics $13,324-340$.

Holmstrom, B., 1999. Managerial incentive problems: a dynamic perspective. Review of Economic Studies 66, 169-182.

Holthausen, R.W., Larcker, D.F., Sloan, R.G., 1995. Annual bonus schemes and the manipulation of earnings. Journal of Accounting and Economics 19, 29-74.

Imhof, L., Kräkel, M., 2011. Winning by a gap. Mimeo.

Innes, R.D., 1990. Limited liability and incentive contracting with ex-ante action choices. Journal of Economic Theory 52, 45-67.

Itoh, H., 2004. Moral hazard and other-regarding preferences. Japanese Economic Review 55, 18-45.

Joseph, K., Kalwani, M.U., 1998. The role of bonus pay in salesforce compensation plans. Industrial Marketing Management 27, 147-159.

Kanemoto, Y., MacLeod, W.B., 1989. Optimal labor contracts with noncontractible human capital. Journal of the Japanese and International Economies 3, 385-402. 
Kanemoto, Y., MacLeod, W.B., 1992. Firm reputation and self-enforcing labor contracts. Journal of the Japanese and International Economies $6,144-162$.

Kim, S.K., 1997. Limited liability and bonus contracts. Journal of Economics and Management Strategy 6, 899-913.

Koch, A.K., Peyrache, E., 2011. Aligning ambition and incentives. Journal of Law, Economics, and Organization 27, 655-688.

Lang, M., 2009. The distinction between first-order and second-order ambiguity aversion. Mimeo.

Lazear, E.P., Rosen, S., 1981. Rank-order tournaments as optimum labor contracts. Journal of Political Economy 89, 841-864.

Malcomson, J.M., 1984. Work incentives, hierarchy, and internal labor markets. Journal of Political Economy 92, 486-507.

Martinez, B., 2011. School 'bonus' plan comes up short. Wall Street Journal, http://online.wsj.com/article/, April 4, 2011.

Miravete, E.J., 2005. The welfare performance of sequential pricing mechanisms. International Economic Review 46, 1321-1361.

Mood, A.M., Graybill, F.A., Boes, D.C., 1974. Introduction to the theory of statistics, third ed. McGraw-Hill, Auckland.

Mookherjee, D., 1984. Optimal incentive schemes with many agents. Review of Economic Studies 51, 433-446.

Murphy, K.J., Oyer, P., 2001. Discretion in executive incentive contracts: Theory and evidence. Mimeo.

Nalebuff, B.J., Stiglitz, J.E., 1983. Prizes and incentives: towards a general theory of compensation and competition. Bell Journal of Economics $14,21-43$. 
Oyer, P., 2000. A theory of sales quotas with limited liability and rent sharing. Journal of Labor Economics 18, 405-426.

Poblete, J., Spulber, D., 2012. The form of incentive contracts: agency with moral hazard, risk neutrality, and limited liability. RAND Journal of Economics 43, 215-234.

Rajan, M.V., Reichelstein, S., 2006. Subjective performance indicators and discretionary bonus pools. Journal of Accounting Research 44, 585618.

Schmitz, P.W., 2005. Workplace surveillance, privacy protection, and efficiency wages. Labour Economics 12, 727-738.

Schöttner, A., 2008. Fixed-prize tournaments versus first-price auctions in innovation contests. Economic Theory 35, 57-71.

Shaked, M., Shanthikumar, J.G., 2007. Stochastic orders. Springer, New York.

Tirole, J., 1988. The theory of industrial organization. MIT Press, Cambridge (Mass.).

Weinschenk, P., 2009. Essays in microeconomics. Dissertation. University of Bonn.

Wolfstetter, E., 1999. Topics in microeconomics. Cambridge University Press, Cambridge. 


\section{Online Appendix}

\section{An Illustrating Example on the Dominance of Bonus Pools}

To illustrate the arguments of Proposition 2 and the magnitude by which the bonus-pool contract may outperform the bonus contract, we consider a specific distribution with strictly increasing hazard rate. We concentrate on the case of $\hat{\gamma}, \gamma \in[0, \bar{\gamma}]$ to condense our arguments. Suppose that the $\theta_{i}(i=1,2)$ are uniformly distributed over $[0,1]$, so that $f(\theta)=1$ and $F(\theta)=\theta$, and let $\bar{\gamma} \in[0,1)$. Furthermore, we assume that $c\left(e_{i}\right)=\kappa e_{i}^{3} / 3$ $(\kappa>0)$ with $\kappa$ being sufficiently large to guarantee interior solutions.

For the optimal bonus contract $\left(\hat{b}_{H}^{*}, 0, \hat{\gamma}^{*}\right)$, the employer maximizes

$$
2 \cdot\left[e_{i}+\frac{1}{2}-\kappa e_{i}^{2}\left(1-\hat{\gamma}+e_{i}\right)\right]
$$

leading to $\hat{\gamma}^{*}=\bar{\gamma}$. The employer implements effort

$$
e^{*}=\frac{1}{3 \kappa}\left(\sqrt{\kappa\left(\kappa(1-\bar{\gamma})^{2}+3\right)}-(1-\bar{\gamma}) \kappa\right)
$$

for both workers via

$$
\hat{b}_{H}^{*}=\frac{1}{9 \kappa}\left(\sqrt{\kappa\left(\kappa(1-\bar{\gamma})^{2}+3\right)}-(1-\bar{\gamma}) \kappa\right)^{2},
$$

yielding expected profit

$$
\Pi^{*}=\frac{4}{27}\left[\frac{1}{\kappa^{2}}\left[\kappa\left(\kappa(1-\bar{\gamma})^{2}+3\right)\right]^{\frac{3}{2}}-(1-\bar{\gamma})\left(\frac{9}{2}+(1-\bar{\gamma})^{2} \kappa\right)\right]+1
$$

which is strictly positive. ${ }^{26}$

In order to compute the optimal bonus-pool contract $\left(b_{H}^{* *}, 0, \gamma^{* *}\right)$, we first have to construct the density $g$. The composed random variable $\delta:=\theta_{1}-\theta_{2}$ can be either negative with $-1 \leq \delta \leq 0$ or positive with $0<\delta \leq 1$. Furthermore, recall that $0 \leq \theta_{1} \leq 1$ and $0 \leq \theta_{2} \leq 1 \Leftrightarrow \delta \leq \theta_{1} \leq 1+\delta$. Thus,

\footnotetext{
${ }^{26} \kappa>1 /[\bar{\gamma}(2+\bar{\gamma})]$ guarantees that $\bar{\gamma}-e^{*} \in(0,1)$.
} 
$0 \leq \theta_{1} \leq 1+\delta$ if $-1 \leq \delta \leq 0$, and $\delta \leq \theta_{1} \leq 1$ if $0<\delta \leq 1$. Applying the convolution formula yields

$$
\begin{gathered}
g(\delta)=\left\{\begin{array}{ccc}
\int_{0}^{1+\delta} f\left(\theta_{1}\right) f\left(\theta_{1}-\delta\right) d \theta_{1} & \text { if } & -1 \leq \delta \leq 0 \\
\int_{\delta}^{1} f\left(\theta_{1}\right) f\left(\theta_{1}-\delta\right) d \theta_{1} & \text { if } & 0<\delta \leq 1 \\
0 & \text { otherwise }
\end{array}\right. \\
=\left\{\begin{array}{ccc}
1+\delta & \text { if } \quad-1 \leq \delta \leq 0 \\
1-\delta & \text { if } & 0<\delta \leq 1 \\
0 & \text { otherwise. }
\end{array}\right.
\end{gathered}
$$

since $f\left(\theta_{1}\right)=1, \forall \theta_{1}$. The cdf $G$ is obtained by integrating $g$ and using the fact that $G(-1)=0$ and $G(1)=1$ :

$$
G(\delta)=\left\{\begin{array}{ccc}
0 & \text { if } & \delta<-1 \\
\delta+\frac{\delta^{2}}{2}+\frac{1}{2} & \text { if } & -1 \leq \delta \leq 0 \\
\delta-\frac{\delta^{2}}{2}+\frac{1}{2} & \text { if } & 0<\delta \leq 1 \\
1 & \text { if } & \delta>1
\end{array}\right.
$$

The employer thus maximizes

$$
2 \cdot\left(e_{i}+\frac{1}{2}-\kappa e_{i}^{2} \frac{1}{2}(1-\gamma)\right)
$$

which gives $\gamma^{* *}=\bar{\gamma}$,

$$
e^{* *}=\frac{1}{(1-\bar{\gamma}) \kappa} \quad \text { and } \quad b_{H}^{* *}=\frac{1}{(1-\bar{\gamma})^{3} \kappa},
$$

leading to expected profit

$$
\Pi^{* *}=\frac{1}{(1-\bar{\gamma}) \kappa}+1
$$

(At the second stage, given $\gamma^{*}=\bar{\gamma}$ and $b_{H}^{*}=1 /\left((1-\bar{\gamma})^{3} \kappa\right)$, worker $i$ 's reaction curve, $e_{i}\left(e_{j}\right)$, is implicitly described by

$$
\kappa e_{i}^{2}=\left\{\begin{array}{lll}
\frac{1}{(1-\bar{\gamma})^{3} \kappa} \cdot\left(1-e_{i}+e_{j}+\bar{\gamma}\right) & \text { if } \quad e_{i}>e_{j}+\bar{\gamma} \\
\frac{1}{(1-\bar{\gamma})^{3} \kappa} \cdot\left(1+e_{i}-e_{j}-\bar{\gamma}\right) & \text { if } \quad e_{i} \leq e_{j}+\bar{\gamma}
\end{array}\right.
$$


which leads to

$e_{i}\left(e_{j}\right)=\left\{\begin{array}{l}\frac{1}{2 \kappa}\left(\sqrt{\frac{1}{(1-\bar{\gamma})^{3} \kappa}\left(\frac{1}{(1-\bar{\gamma})^{3} \kappa}+4 \kappa\left(1+\bar{\gamma}+e_{j}\right)\right)}-\frac{1}{(1-\bar{\gamma})^{3} \kappa}\right) \quad \text { if } \quad e_{i}>e_{j}+\bar{\gamma} \\ \frac{1}{2 \kappa}\left(\sqrt{\frac{1}{(1-\bar{\gamma})^{3} \kappa}\left(\frac{1}{(1-\bar{\gamma})^{3} \kappa}+4 \kappa\left(1-\bar{\gamma}-e_{j}\right)\right)}+\frac{1}{(1-\bar{\gamma})^{3} \kappa}\right) \quad \text { if } \quad e_{i} \leq e_{j}+\bar{\gamma}\end{array}\right.$

In the upper case, $e_{i}\left(e_{j}\right)$ and $e_{i}=e_{j}+\bar{\gamma}$ intersect at $\tilde{e}_{j}=\frac{1}{\kappa} \sqrt{\frac{1}{(1-\bar{\gamma})^{3}}}-\bar{\gamma}$. In the lower case, an intersection between $e_{i}\left(e_{j}\right)$ and $e_{i}=e_{j}+\bar{\gamma}$ will exist if and only if

$$
\sqrt{\frac{1}{(1-\bar{\gamma})^{3} \kappa}\left(\frac{1}{(1-\bar{\gamma})^{3} \kappa}+4 \kappa\left(1-\left(\bar{\gamma}+e_{j}\right)\right)\right)}=2 \kappa\left(e_{j}+\bar{\gamma}\right)-\frac{1}{(1-\bar{\gamma})^{3} \kappa}>0 .
$$

In addition, we need

$$
\frac{1}{(1-\bar{\gamma})^{3} \kappa}+4 \kappa\left(1-\left(\bar{\gamma}+e_{j}\right)\right) \geq 0
$$

Combining both conditions yields

$$
\frac{1}{2(1-\bar{\gamma})^{3} \kappa^{2}}<\bar{\gamma}+e_{j} \leq \frac{1}{4(1-\bar{\gamma})^{3} \kappa^{2}}+1
$$

which requires that $\kappa>1 /\left[2(1-\bar{\gamma})^{\frac{3}{2}}\right]$. Condition (16) is satisfied for $e_{j}=e^{* *}$ if $\kappa$ is sufficiently large. Thus, we assume $\kappa$ to be sufficiently large so that an intersection is guaranteed. Solving $e_{i}\left(e_{j}\right)=e_{j}+\bar{\gamma}$ (described by (14)) for $e_{j}$ gives $e_{j}=\frac{1}{\kappa} \sqrt{\frac{1}{(1-\bar{\gamma})^{3}}}-\bar{\gamma} \equiv \tilde{e}_{j}$.

To sum up, the reaction curve $e_{i}\left(e_{j}\right)$ starts with a positive slope at $e_{j}=0$ (see the upper case). It intersects with $e_{i}=e_{j}+\bar{\gamma}$ at $e_{j}=\tilde{e}_{j}$ and then proceeds with a negative slope (see the lower case). ${ }^{27}$ The reaction curve ends where condition (15) holds with equality (i.e., at $e_{j}=\frac{1}{4(1-\bar{\gamma})^{3} \kappa^{2}}+1-\bar{\gamma}$ ). Mirroring

\footnotetext{
${ }^{27}$ The shape of the workers' reaction curves is typical of contest or relative-compensation games. First, it reflects strategic complements and then switches to strategic substitutes. See, e.g., Wärneryd [Games and Economic Behavior, vol. 33 (2000), 145-158]; Yildirim [Games and Economic Behavior, vol. 51 (2005), 213-227]; Konrad [Strategy and Dynamics in Contests. 2009. Oxford University Press, New York], p. 46.
} 
$e_{i}\left(e_{j}\right)$ against the 45-degree line gives worker $j$ 's reaction curve, $e_{j}\left(e_{i}\right)$. Both reaction curves intersect in the symmetric equilibrium with $e_{i}=e_{j}=e^{* *}$.)

Comparing the solution under the optimal individual bonus contracts with the solution under the optimal bonus-pool contract leads to the following results:

Example Suppose noise is uniformly distributed over $[0,1]$ and workers have cubic costs $c(e)=\kappa e^{3} / 3$. Then $r(\alpha)=2 \cdot r_{\theta}(\alpha), \forall \alpha>0$, and we obtain $b_{H}^{* *}>4 \hat{b}_{H}^{*}$ and $e^{* *}>2 e^{*}$. Moreover, $\Pi^{* *}>\Pi^{*}$. The probability that both $\hat{b}_{H}^{*}$ are paid out under the optimal bonus contract, $\left(1-F\left(\bar{\gamma}-e^{*}\right)\right)^{2}$, is larger than the probability that $b_{H}^{* *}$ is paid out under the optimal bonus-pool contract, $2 \cdot(1-G(\bar{\gamma}))$.

Proof Using the expressions for the optimal effort levels, we have

$$
e^{* *}-2 e^{*}=\frac{3+2 \kappa(1-\bar{\gamma})^{2}-2(1-\bar{\gamma}) \sqrt{\kappa^{2}(1-\bar{\gamma})^{2}+3 \kappa}}{3 \kappa(1-\bar{\gamma})}>0,
$$

showing that $e^{* *}>2 e^{*}$. Hence, by (7) and (4),

$$
4 \hat{b}_{H}^{*}=\kappa\left(2 e^{*}\right)^{2}<\kappa\left(e^{* *}\right)^{2}=b_{H}^{* *} g(\bar{\gamma})<b_{H}^{* *} .
$$

To prove that $\Pi^{* *}>\Pi^{*}$, we only have to compare

$$
\Pi^{*}=2 \cdot\left(e_{i}+\frac{1}{2}-\kappa e_{i}^{2}\left(1-\bar{\gamma}+e_{i}\right)\right)
$$

with

$$
\Pi^{* *}=2 \cdot\left(e_{i}+\frac{1}{2}-\kappa e_{i}^{2} \frac{1}{2}(1-\bar{\gamma})\right) .
$$

The two expressions show that under a bonus-pool contract the employer can always implement the same effort level at lower cost compared to the bonus contract. As $e^{* *}>2 e^{*}$ points out, the employer is even better off by implementing much more effort under a bonus-pool contract. 
We have

$$
\begin{aligned}
2 \cdot(1-G(\bar{\gamma})) & =(1-\bar{\gamma})^{2} \\
& =\frac{1}{9 \kappa}\left(5 \kappa(1-\bar{\gamma})^{2}\right)+\frac{4}{9 \kappa}(1-\bar{\gamma}) \sqrt{\kappa\left(\kappa(1-\bar{\gamma})^{2}\right)} \\
& <\frac{1}{9 \kappa}\left(5 \kappa(1-\bar{\gamma})^{2}+3\right)+\frac{4}{9 \kappa}(1-\bar{\gamma}) \sqrt{\kappa\left(\kappa(1-\bar{\gamma})^{2}+3\right)} \\
& =\left(1-F\left(\bar{\gamma}-e^{*}\right)\right)^{2} .
\end{aligned}
$$

The example shows that the major idea of creating incentives via a bonus contract and a bonus-pool contract is very similar. In both cases, the employer chooses a large threshold to minimize expected implementation costs. In order to restore worker motivation, the employer has to combine this quota with a sufficiently high bonus payment. Under the optimal bonus-pool contract, incentive pay is considerably higher than under individual bonus contracts (i.e., $b_{H}^{* *}>4 \hat{b}_{H}^{*}$ ). Since the production technology is the same under either contract but expected implementation costs are lower under the bonus pool due to $r(\alpha)=2 \cdot r_{\theta}(\alpha), \forall \alpha>0$, the optimal bonus pool yields implemented effort that is more than twice as high as that under the optimal bonus contract $\left(e^{* *}>2 e^{*}\right)$.

When choosing the optimal threshold for the bonus pool, the employer faces the following trade-off: A large threshold minimizes (i) the probability of paying out the bonus pool (implementation-cost effect) but also (ii) the workers' incentives, since $g(\bar{\gamma})$ decreases in $\bar{\gamma}>0$ (incentive effect). However, this trade-off is absent under a bonus contract in this example since $f$ is a constant so that a large quota does not influence workers' incentives. Consequently, the employer chooses the maximum possible quota under the optimal bonus contract. He chooses the same threshold under the optimal bonus-pool contract because the implementation-cost effect strictly dominates the incentive effect. Despite the trade-off, the optimal bonus-pool contract outperforms the optimal bonus contract, which is indicated by the large implemented effort and the relatively low probability of paying out the bonus pool (i.e., $\left.\left(1-F\left(\bar{\gamma}-e^{*}\right)\right)^{2}>2 \cdot(1-G(\bar{\gamma}))\right){ }^{28}$

\footnotetext{
${ }^{28}$ We have disregarded the fact that, under the optimal bonus contract, the employer
} 
The comparison of the profits clearly shows the superiority of the bonus pool (i.e., $\Pi^{* *}>\Pi^{*}$ ). Inserting numbers for the parameters of the example demonstrates the magnitude of this superiority. If, for example, $\bar{\gamma}=0.3$ and $\kappa=1.5$, then $\Pi^{*} \approx 1.33$ and $\Pi^{* *} \approx 1.95$, that is, the optimal bonuspool contract is more profitable than the optimal bonus contract by about 47 percent. If $\bar{\gamma}=0.5$ and $\kappa=2.7$, then $\Pi^{*} \approx 1.25$ and $\Pi^{* *} \approx 1.74$ and, hence, the bonus-pool contract still outperforms the bonus contract by about 39 percent. These examples point out that switching from the optimal bonus contract to the optimal bonus-pool contract can increase an employer's expected profits considerably. ${ }^{29}$

\section{Parameterized Distribution Formulation}

We consider a scenario in which the employer has only limited capital $K$ and show that a bonus-pool contract can again strictly outperform individual bonus contracts.

Let the two workers choose binary efforts $e \in\left\{e_{L}, e_{H}\right\}$ with $e_{L}<e_{H}$. The corresponding effort costs are $c\left(e_{L}\right)=0<c:=c\left(e_{H}\right)$. We assume that it always pays for the employer to implement $e_{H}$ instead of $e_{L} \cdot{ }^{30}$ The verifiable performance measure $x_{i}$ follows a probability distribution that depends on the worker's effort choice. Let $P\left(x_{j} \mid e_{k}\right)=P_{j k}$ with $j=1, \ldots, m$ and $k=$ $L, H$ denote the probability that the performance measure of a worker leads to realization $x_{j}$ given that the worker chose effort $e_{k}$. We assume that $x_{1}<x_{2}<\cdots<x_{m}$ and $P_{j H}<P_{j L}$ for all $j \neq m$, and $P_{m H}>P_{m L}$ so has to pay out one of the two bonuses with probability $2 F\left(\bar{\gamma}-e^{*}\right)\left[1-F\left(\bar{\gamma}-e^{*}\right)\right]$, which even strengthens our argument.

${ }^{29}$ For the case of an increasing hazard rate, Poblete and Spulber (2012, Proposition 2) suggest to compensate each worker according to $\max \left\{x_{i}\left(e_{i}\right)-r, 0\right\}$ for some $r \geq 0$. We can show for our example that the optimal bonus-pool contract also leads to higher profits compared to this contract.

${ }^{30}$ This simplifying assumption is often made to get rid of further cases that do not really add to the analysis. 
that the distribution $P\left(x_{j} \mid e_{H}\right)$ dominates the distribution $P\left(x_{j} \mid e_{L}\right)$ within the meaning of first-order stochastic dominance. ${ }^{31}$ As before, we assume that workers are protected by limited liability (i.e., negative wages are not feasible) and that each worker has a zero reservation value so that we can ignore the workers' participation constraint in the following. To sum up, in the given setting the employer wants to choose the incentive scheme that implements $e_{H}$ at minimal costs.

We start with the analysis of the optimal individual bonus contracts. Since both workers are identical, we can focus on the decision problem of one of them. Let $\hat{b}_{j} \geq 0$ denote the bonus payment to a worker if his performance measure takes the value $x_{j}(j=1, \ldots, m)$. The incentive constraint for implementing $e_{H}$ reads as ${ }^{32}$

$$
\sum_{j=1}^{m} \hat{b}_{j} \cdot P_{j H}-c \geq \sum_{j=1}^{m} \hat{b}_{j} \cdot P_{j L} \Leftrightarrow \sum_{j=1}^{m} \hat{b}_{j}\left(P_{j H}-P_{j L}\right) \geq c .
$$

Hence, the optimal individual bonus contract $\left(\hat{b}_{1}^{*}, \ldots, \hat{b}_{m}^{*}\right)$ that implements $e_{H}$ at lowest costs is described by

$$
\hat{b}_{j}^{*}=0 \text { for all } j \neq m, \quad \text { and } \quad \hat{b}_{m}^{*}=\frac{c}{P_{m H}-P_{m L}} .
$$

The maximal payment to both workers, $2 \hat{b}_{m}^{*}$, is not allowed to exceed the employer's capital, $K$, so that the optimal individual bonus contract will only be feasible if the employer's limited-liability condition

$$
\frac{c}{P_{m H}-P_{m L}} \leq \frac{K}{2}
$$

is satisfied.

If the employer is allowed to pool the bonus payments for the two workers, individual payments can be made contingent on the realizations of both workers' performance measures. Again, we can focus on the situation of one

\footnotetext{
${ }^{31}$ Skipping the assumption $P_{j H}<P_{j L}, \forall j \neq m$, would only strengthen our results.

${ }^{32} \mathrm{As}$ usual we assume that the worker chooses the higher effort level when being indifferent between $e_{H}$ and $e_{L}$.
} 
of the workers. Let $b_{i j}$ denote the bonus payment to a worker if his performance measure has the realization $x_{i}$ and that of his co-worker the realization $x_{j}, i, j \in\{1, \ldots, m\}$. The incentive constraint for implementing $e_{H}$ for each worker - i.e., the Nash equilibrium condition that the worker does not want to deviate from $e_{H}$ given that his co-worker chooses $e_{H}$ - is given by

$$
\sum_{i=1}^{m}\left(\left(P_{i H}-P_{i L}\right) \sum_{j=1}^{m} b_{i j} \cdot P_{j H}\right) \geq c .
$$

Thus, a bonus-pool contract given by $\left(b_{i j}\right)$ leads to high effort by each worker at minimal expected costs if and only if $b_{i j}=0$ for all $i<m$ and all $j$, and

$$
\sum_{j=1}^{m} b_{m j} \cdot P_{j H}=\frac{c}{P_{m H}-P_{m L}} .
$$

A wealth-constrained employer must be able to pay the bonuses $b_{m j}$ to the workers under any pair of realizations $x_{i}$ and $x_{j}$. Even in the worst case the employer must have enough capital to pay the respective bonuses. Hence, to minimize the maximal possible cost, it is optimal to choose $b_{m j}^{*}$ such that total labor costs are identical for any pair $\left(x_{i}, x_{j}\right)$. The bonus $b_{m m}^{*}$ has to be paid twice if both workers' performance measures take the highest realization $x_{m}$, whereas in all other situations we have at most one positive bonus payment. It is, therefore, optimal for the employer to choose, for some $b^{*}, b_{m j}^{*}=b^{*}$ for all $j \neq m$, and $b_{m m}^{*}=\frac{1}{2} b^{*}$ at the left-hand side of equation (18), leading to

$$
\sum_{j=1}^{m-1} b^{*} \cdot P_{j H}+\frac{1}{2} b^{*} \cdot P_{m H}=\frac{c}{P_{m H}-P_{m L}} \Leftrightarrow b^{*}=\frac{c}{\left(P_{m H}-P_{m L}\right)\left(1-\frac{1}{2} P_{m H}\right)} .
$$

This optimal bonus pool will only be feasible under a wealth-constrained employer if

$$
b^{*} \leq K \Leftrightarrow \frac{c}{P_{m H}-P_{m L}} \leq K\left(1-\frac{1}{2} P_{m H}\right)
$$

The comparison of (17) and (19) shows that $1-\frac{1}{2} P_{m H}>\frac{1}{2} \Leftrightarrow P_{m H}<1$ is true. Hence, there exist values of the employer's capital $K$ for which the optimal bonus-pool contract is feasible and implements $e_{H}$ whereas the optimal individual bonus contract is not feasible, but the opposite case that the 
individual bonus contract is feasible and the bonus-pool contract is infeasible can never happen. ${ }^{33}$

\footnotetext{
${ }^{33}$ The employer's expected labor costs are identical under both contracts as the two incentive constraints are binding.
} 